\title{
A NOTE ON TORSION-FREE NIL GROUPS
}

\author{
RIMHAK REE AND ROBERT J. WISNER
}

Introduction. A nil group is an (additively written) abelian group $G$ such that every associative ring $R$ with $G$ as its additive group has trivial multiplication, i.e., $x y=0$ for every $x, y \in R$. Szele [2] has shown that every nil group is either a torsion group (every element of finite order) or a torsion-free group (every element except the identity of infinite order), and he has completely characterized the torsion nil groups. In [3], Szele discusses some questions related to nil groups, but is unable to find conditions for the existence of torsionfree nil groups.

An additive group $G$ is said to be strongly nil if there is no nontrivial ring, associative or nonassociative, which has $G$ as its additive group. It is the purpose of this note to characterize those nil and strongly nil groups which are (isomorphic to) a weak direct sum of subgroups of the additive group of rationals, and to show that every such nil group is also strongly nil.

The authors would like to express thanks to the referee for helpful suggestions.

The basic lemma. We use, in modified form, the characterization of the subgroups of the additive rationals $R^{+}$given by Beaumont and Zuckerman [1]: designate by $p_{1}, p_{2}, \cdots, p_{j}, \cdots$ the primes in their natural order; then for any subgroup $K$ of $R^{+}$which contains the rational integers, let $k_{j}$ be the greatest non-negative integer $n$ such that $p_{j}^{-n}$ appears in $K$, if such an integer exists, and the symbol $\infty$ otherwise. $K$ is, then, the set of all rationals $u / v$ with $u$ an arbitrary integer and $v$ an arbitrary integer of the form $\prod_{j} p_{j}^{n_{j}}$ with $n_{j} \leqq k_{j}$. We denote $K$ by the sequence $\left(k_{1}, k_{2}, \cdots, k_{j}, \cdots\right)$; and since every nonzero subgroup of $R^{+}$is isomorphic to one containing the rational integers, this characterization is essentially complete. The initials i.c. will designate "integer containing" with reference to subgroups of $R^{+}$.

If $K=\left(k_{1}, k_{2}, \cdots\right), L=\left(l_{1}, l_{2}, \cdots\right), M=\left(m_{1}, m_{2}, \cdots\right)$ are i.c. subgroups of $R^{+}$, define $(K, L, M)$ to be the set of all rationals $t$ such that $t K L \subset M$. It is clear that $(K, L, M)$ is a subgroup of $M$, and we shall

Presented to the Society, May 1, 1954; received by the editors September 14, 1954 and, in revised form, April 13, 1955. 
need conditions making $(K, L, M)=0$. Assume $(K, L, M) \neq 0$, then

(1) if $k_{j}=\infty$, then $m_{j}=\infty$,

(2) if $l_{j}=\infty$, then $m_{j}=\infty$,

(3) if $k_{j}$ and $l_{j}$ are both finite, then $m_{j} \geqq k_{j}+l_{j}$ for almost all $j$.

For if $k_{j}=\infty$ and $m_{j}<\infty$, every element of $(K, L, M)$ would be divisible by $p_{j}^{n}$ for arbitrarily high powers $n$, and this is a contradiction unless $(K, L, M)=0$. The proof of (2) is analogous. If $k_{j}<\infty$ and $l_{j}<\infty$, and if there were infinitely many subscripts $j$ for which $m_{j}<k_{j}+l_{j}$, every element of $(K, L, M)$ would have to be divisible by infinitely many primes, yielding again $(K, L, M)=0$. This proves (3).

On the other hand, if (1), (2), (3) hold, and if $m_{j} \geqq k_{j}+l_{j}$ for all $j$, then $(K, L, M)$ contains 1 . Otherwise, if $j_{1}, \cdots, j_{q}$ denote those subscripts for which $k_{j_{i}}<\infty, l_{j_{i}}<\infty, m_{j_{i}}<k_{j_{i}}+l_{j_{i}}, i=1, \cdots, q$, then

$$
\prod_{i=1}^{q} p_{i_{i}}^{n_{i}} \in(K, L, M)
$$

where $n_{i}=k_{j_{i}}+l_{j_{i}}-m_{j_{i}}$, and again $(K, L, M) \neq 0$.

LEMMA. $(K, L, M)=0$ if, and only if, (1) or (2) or (3) is false.

(As an easy application of this lemma, suppose $K$ is an i.c. nil subgroup of $R^{+}$. Then $(K, K, K)=0$, and since (1) and (2) are satisfied, (3) must be false, i.e., $k_{j}<2 k_{j}$ for an infinite number of subscripts $j$. Hence, the i.c. nil subgroups of $R^{+}$are given by those sequences $\left(k_{1}, k_{2}, \cdots\right)$ for which $0<k_{j}<\infty$ for an infinite number of $k_{j}$ 's. This is essentially Theorem 3 of [1].)

Direct sum case. Let $\Lambda$ be any index set, and let $\left\{H_{\lambda}\right\}, \lambda \in \Lambda$, be a set of i.c. subgroups of $R^{+}$. The weak direct sum $G$ of these groups $H_{\lambda}$ is defined in terms of a basis $\left\{a_{\lambda}\right\}, a_{\lambda} \in H_{\lambda}$, as the set of all elements

$$
x=\sum_{\lambda} r_{\lambda}(x) a_{\lambda}
$$

where: $r_{\lambda}(x) \in H_{\lambda}$; for each $x, r_{\lambda}(x)=0$ for almost all $\lambda \in \Lambda$; equality and addition are defined componentwise; $0 a_{\lambda}=0$ and $1 a_{\lambda}=a_{\lambda}$ for all $\lambda \in \Lambda$. If $R$ is a ring with $G$ as its additive group, then

$$
a_{\lambda} a_{\mu}=\sum_{\nu} s_{\lambda_{\mu}}^{\prime} a_{\nu} \in R
$$

for suitable structure constants $s_{\lambda_{\mu}}^{\nu} \in H_{\nu}$, and

$$
r_{\nu}(x y)=\sum_{\lambda, \mu} r_{\lambda}(x) r_{\mu}(y) s_{\lambda \mu}^{\nu}
$$


THEOREM 1. G is strongly nil if and only if $\left(H_{\lambda}, H_{\mu}, H_{\nu}\right)=0$ for every $\lambda, \mu, \nu$ in $\Lambda$.

TheOREM 2. $G$ is nil if and only if $\left(H_{\lambda}, H_{\mu}, H_{\nu}\right)=0$ for every $\lambda, \mu, \nu$ in $\Lambda$ satisfying either (i) $\lambda=\mu=\nu$ or (ii) $\lambda \neq \nu, \mu \neq \nu$.

Proofs. Assume $t \neq 0$ is an element of $\left(H_{\lambda}, H_{\mu}, H_{\nu}\right)$ for some $\lambda, \mu, \nu$ of $\Lambda$. For each pair of elements $x, y \in G$, define multiplication by

$$
x y=\operatorname{tr}_{\lambda}(x) r_{\mu}(y) a_{\nu}
$$

and this makes $G$ into a nontrivial ring $R$ (since $a_{\lambda} a_{\mu} \neq 0$ ). Associativity of this multiplication is implied by (i) or (ii):

$$
\begin{aligned}
& (x y) z=\operatorname{tr}_{\lambda}\left(\operatorname{tr}_{\lambda}(x) r_{\mu}(y) a_{\nu}\right) r_{\mu}(z) a_{\nu}, \\
& x(y z)=\operatorname{tr}_{\lambda}(x) r_{\mu}\left(\operatorname{tr}_{\lambda}(y) r_{\mu}(z) a_{\nu}\right) a_{\nu} .
\end{aligned}
$$

If $\lambda=\mu=\nu$, each of these becomes $t^{2} r_{\lambda}(x) r_{\lambda}(y) r_{\lambda}(z) a_{\lambda}$. If $\lambda \neq \nu, \mu \neq \nu$, $r_{\lambda}\left(\operatorname{tr}_{\lambda}(x) r_{\mu}(y) a_{\nu}\right)=r_{\mu}\left(\operatorname{tr}_{\lambda}(y) r_{\mu}(z) a_{\nu}\right)=0$.

Conversely, let $R$ be a ring over $G$, and assume $\left(H_{\lambda}, H_{\mu}, H_{\nu}\right)=0$ for some $\lambda, \mu, \nu$. It is clear that $s_{\lambda \mu}^{\nu}=0$, then, from which it follows that $G$ is strongly nil. However, if $s_{\lambda \mu}^{\nu}=0$ under conditions (i) or (ii), then $a_{\lambda} a_{\lambda}=0$ for every $\lambda$, and $a_{\lambda} a_{\mu}=s_{\lambda \mu}^{\lambda} a_{\lambda}+s_{\lambda \mu}^{\mu} a_{\mu}$. The associative law imposed on the product $\left(a_{\lambda} a_{\mu}\right) a_{\mu}$ yields $s_{\lambda_{\mu}}^{\mu}=0$, and a similar argument on the product $a_{\lambda}\left(a_{\lambda} a_{\mu}\right)$ yields $s_{\lambda \mu}^{\mu}=0$. Therefore, $G$ is nil.

Remark. It is interesting that for groups which are the weak direct sum of i.c. subgroups of $R^{+}$, "nil" implies "strongly nil." For suppose $t$ is a nonzero element of $(K, L, K)$. Then $t K L \subset K$, and so $t K L^{2} \subset K L$, whence $t^{2} K L^{2} \subset t K L \subset K$. It follows that $t^{2}$ is an element of $(L, L, K)$. Similarly, $(K, L, L) \neq 0$ implies $(K, K, L) \neq 0$. Thus, the conditions of Theorem 2 imply those of Theorem 1.

\section{REFERENCES}

1. R. A. Beaumont and H. S. Zuckerman, A characterization of the subgroups of the additive rationals, Pacific Journal of Mathematics vol 1 (1951) pp. 169-177.

2. T. Szele, Zur Theorie der Zeroringe, Math. Ann. vol. 121 (1949) pp. 242-246.

3. - Gruppentheoretische Beziehungen bei gewissen Ringkonstruktionen, Math. Zeit. vol. 54 (1951) pp. 168-180.

University OF BRITISH Columbia AND HAVERFoRd COLlege 